\title{
Double-layered purse string uterine suture employing the French ambulatory cesarean section technique compared with single-layer continuous uterine suture: An ultrasound evaluation randomized trial.
}

kaouther dimassi ${ }^{1}$, olivier $\mathrm{ami}^{2}$, rania merrai ${ }^{3}$, luka velemir ${ }^{4}$, benedicte $\operatorname{simon}^{5}$, denis fauck $^{6}$, and amel triki ${ }^{3}$

${ }^{1}$ University of Tunis El Manar Faculty of Medicine of Tunis

${ }^{2}$ Ramsay Healthcare France, La Muette, Paris, France

${ }^{3}$ University of Tunis El Manar

${ }^{4}$ Lenval foundation Polyclinique Santa Maria, Nice, France.

${ }^{5}$ Ramsay Healthcare France, Les Franciscaines, Versailles, France.

${ }^{6}$ Ramsay Healthcare France, Saint Lambert, La Garenne-Colombes, France

February 22, 2021

\begin{abstract}
Objective: To test the hypothesis that compared to single layer continuous uterine suture (SLCUS), a double layered purse string uterine suture (PSUS) significantly reduces cesarean scar defect (CSD) rates, without increasing the perioperative maternal morbidity. Design : Interventional prospective, randomized study . Setting: University obstetric units in Tunisia. Population: 100 pregnant women with an indication of a planned Caesarean. Methods: Patients were enrolled in 2 groups according to the uterine suture technique: SLCUS or PSUS. A Saline infusion hysterosonography was performed by the same senior obstetrician blinded to the uterine suture technique 6 months after surgery. Main Outcome measures: Operative time and Calculated blood loss (CBL) were used for the short time analysis. Uterine and CSD measurements were used for the mid time analysis . Results : Despite a few minutes longer operative time in SUS group ( $7.17 \pm 2.31 \mathrm{~min}$ Vs. $6.31 \pm 3.04 \mathrm{~min}, \mathrm{p}=0.028$ in SLCUS group; $\mathrm{p}<10-3)$; there was no significant difference in terms of CBL $(520+-58$ in SUS group vs. $536+-50$ ml in SLCUS group, $\mathrm{p}=$ 0.724). The medium-term analysis showed a significant decrease in the rate of CSD with the PSUS: $6.66 \%$ vs.40\% with SLUCS; $\mathrm{p}=0.002$. Moreover, SLUCS was the leading risk factor for CSD : adjusted OR=6;95\% CI [0- 1],p < 10-3) . Conclusion : Compared to single layer continuous suture, purse string uterine suture significantly reduces cesarean scar defect rates, without increasing the perioperative maternal morbidity. NCT03930134. https://clinicaltrials.gov/ct2/show/NCT03930134
\end{abstract}

\section{Hosted file}

manuscript purse string BJOG.pdf available at https://authorea.com/users/397352/articles/ 510252-double-layered-purse-string-uterine-suture-employing-the-french-ambulatorycesarean-section-technique-compared-with-single-layer-continuous-uterine-suture-anultrasound-evaluation-randomized-trial 

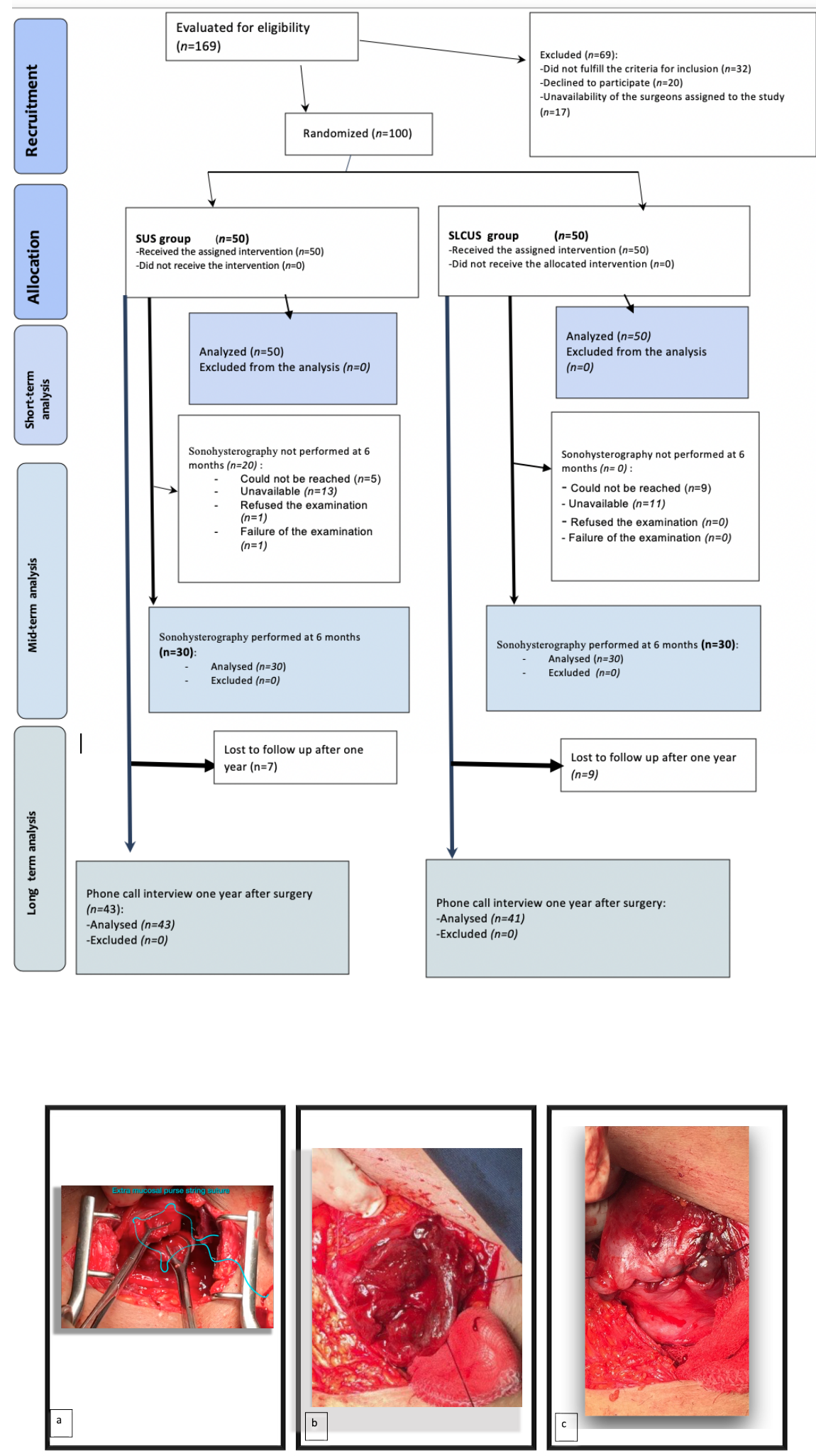

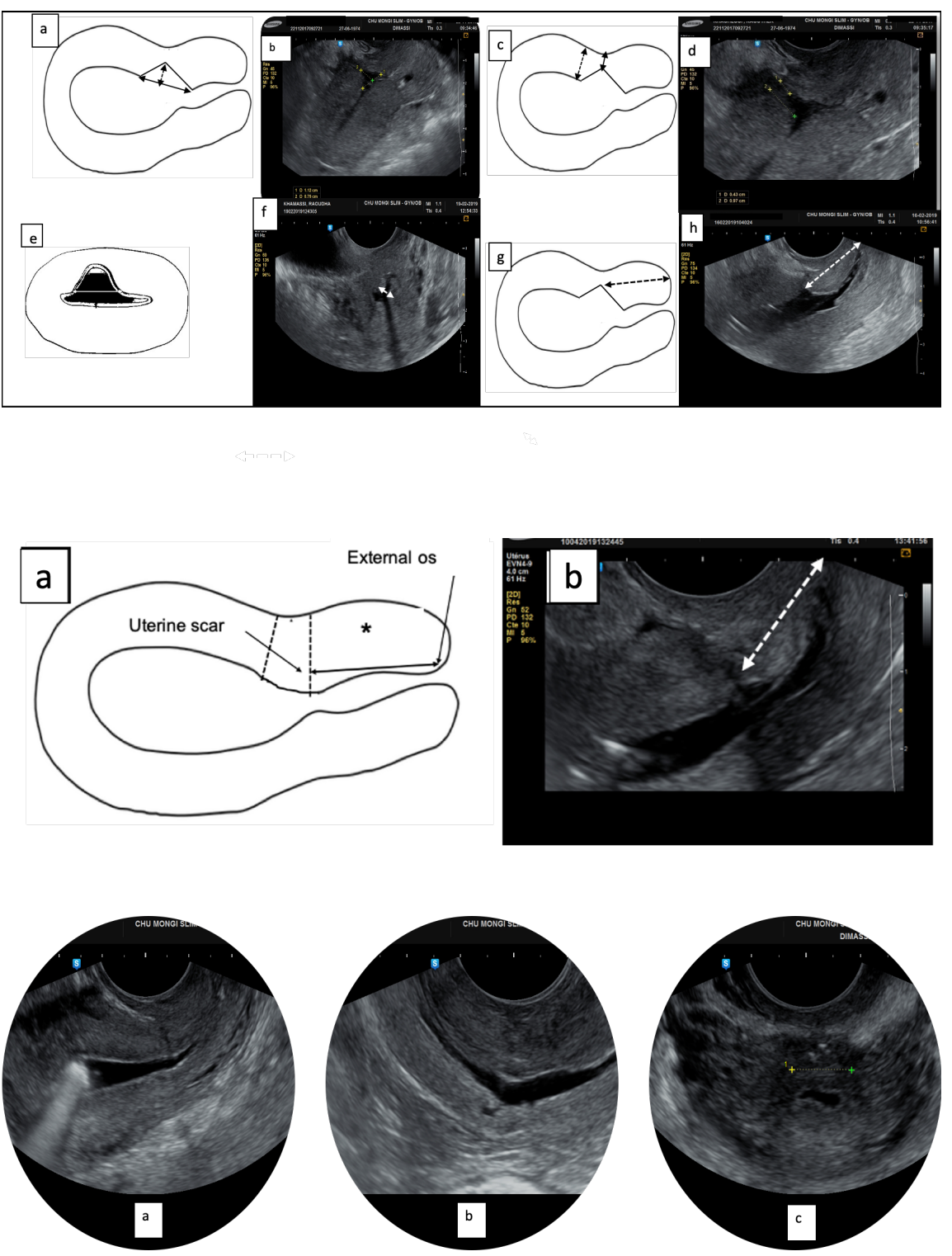